1

2

\title{
Synthesis and Neurotrophic Activity Studies of Illicium Sesquiterpene Natural Product Analogs
}

\author{
Johannes Richers, ${ }^{[a]}$ Alexander Pöthig ${ }^{[b]}$ Eberhardt Herdtweck, ${ }^{[b]}$ Claudia Sippel, ${ }^{[c]}$ Felix Hausch, ${ }^{[d]}$ and \\ Konrad Tiefenbacher ${ }^{[e, f] *}$
}

\begin{abstract}
Neurotrophic natural products hold potential as privileged structures for the development of therapeutic agents against neurodegeneration. However, only few studies have been conducted in order to investigate a common pharmacophoric motif and the structure-activity relationship. Here, an investigation of structurally more simple analogs of neurotrophic sesquiterpenes of the illicium family is presented. A concise synthetic route enables the preparation of the carbon framework of $( \pm)$-Merrilactone $A$ and $( \pm)$-Anislactone $A / B$ in gram-scale. Thereby providing access to a series of structural analogs by modification of the core structure, including variation of oxidation levels and alteration of functional groups. In total, 15 derivatives of the natural products have been synthesized and tested for their neurite outgrowth activity. Our studies indicate that the promising biological activity can be retained by structurally more simple natural product analogs, which are accessible by a straightforward synthetic route.
\end{abstract}

\section{Introduction}

The awe-inspiring structural diversity of natural products has always motivated organic chemists to investigate and develop new synthetic routes, methods and applications. Significant discoveries have been made by studying the chemical structure of these compound and their properties. In particular, the investigation of promising biological activities has resulted in an

[a] Johannes Richers M.Sc.

Department Chemistry

Technical University of Munich

Lichtenbergstraße 4, 85747 Garching, Germany

[b] Dr. Alexander Pöthig, Dr. Eberhardt Herdtweck

Catalysis Research Center

Technical University of Munich

Ernst-Otto-Fischer-Str. 1, 85747 Garching, Germany

[c] Claudia Sippel

Department of Translational Research in Psychiatry

Max Planck Institute of Psychiatry

Kraepelinstr. 2-10, 80804 Munich, Germany

[d] Prof. Dr. Felix Hausch

Clemens-Schöpf-Institute of Organic Chemistry and Biochemistry

Technische Universität Darmstadt

Alarich-Weiss-Str. 4, 64287 Darmstadt, Germany

[e] Prof. Dr. Konrad Tiefenbacher*

Department of Chemistry

University of Basel

St. Johanns-Ring 19, 4056 Basel, Switzerland

E-mail: konrad.tiefenbacher@unibas.ch

[f] Department of Biosystems Science and Engineering

ETH Zurich

Mattenstrasse 26, 4058 Basel, Switzerland

E-mail: tkonrad@ethz.ch

Supporting information for this article can be found under:

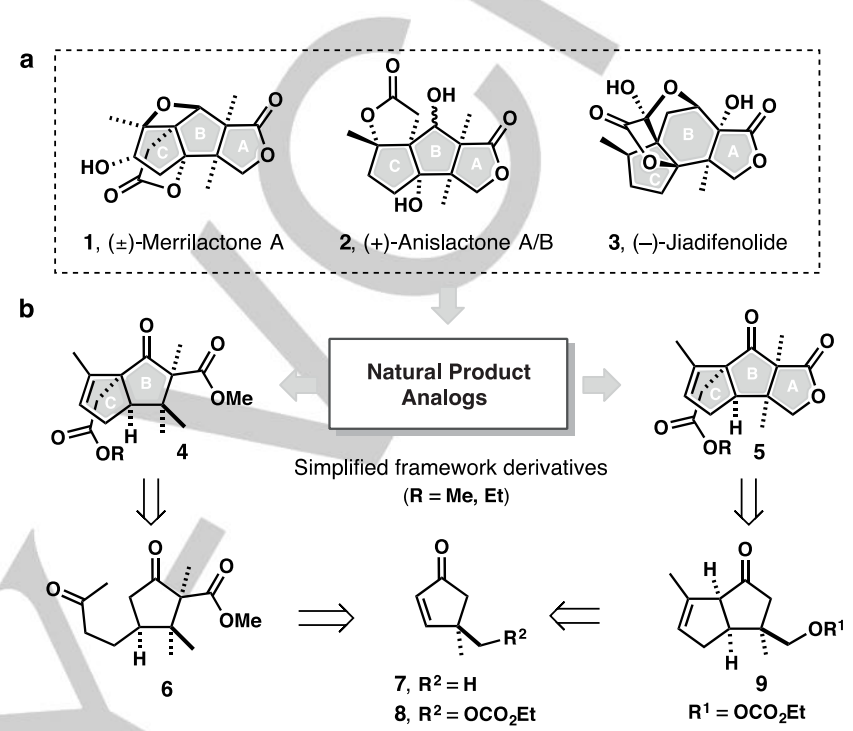

Figure 1. a Neurotrophic sesquiterpene natural products with their core structure (marked in grey). b Natural product analogs and retrosynthesis.

array of applications of natural products and their analogs as pharmaceuticals. Therefore, natural products are often used as a starting point for drug discovery, in which lead compounds undergo extensive optimization and structural variation. However, the complexity of natural products can often only be constructed via lengthy synthetic routes which may hamper drug discovery. One approach to circumvent this issue is to reduce the complexity of the natural products, while retaining the desired biological activity. Such strategies have been recently summarized by Gademann and co-workers who described the promising role of natural product based fragments in drug discovery. They highlighted examples where the structural complexity of natural products was reduced successfully. ${ }^{[1]}$ Structurally more simple analogues, accessible via shorter synthetic routes, can greatly improve chemical tractability and can therefore overcome the limitations of lengthy chemical syntheses.

Since the discovery of several neurotrophic natural products which form part of the illicium family, e.g. Merrilactone A (1), ${ }^{[2]}$ Anislactone A/B (2), ${ }^{[3]}$ and Jiadifenolide $(3)^{[4]}$ (Figure 1a), these compounds have received remarkable attention from the synthetic community, ${ }^{[5]}$ not least because of their challenging chemical structure and their promising biological activity. ${ }^{[2,4,6]}$ The polycyclic small molecules are capable of promoting outgrowth in neuronal cultures. ${ }^{[7]}$ Therefore, these privileged structures are considered to hold potential for the development of pharmaceuticals for the treatment of neurodegenerative diseases such as Parkinson's or Alzheimer's (AD). ${ }^{[8]}$ In contrast to protein neurotrophins, ${ }^{\left[{ }^{[9]}\right.}$ e.g. nerve growth factor (NGF) or brain-derived neurotrophic factor (BNDF), small-molecule neurotrophin-like 
compounds have been investigated because of their desirable pharmacokinetic properties and pharmacological advantages, i.e. low molecular weight, high serum stability and most importantly blood-brain-barrier permeability. ${ }^{[10]}$ In the case of majucin-type sesquiterpenes, such as Jiadifenolide (2), several studies were conducted in order to determine a common pharmacophoric motif. ${ }^{[6 a, c]}$ These investigations led to the identification of several potent analogs and therefore have shown that certain synthetic derivatives can have activities comparable to the natural product.

Although a great number of synthetic studies on Merrilactone A (1) and Anislactone A/B (2) have been performed, no systematic investigation on their structure-activity relationship is available. This might be due to the length of the syntheses reported. As a consequence, variations of the carbon frameworks of the natural products are not readily accessible. We realized that in order to overcome this limitation a synthetic route, which provides simple access to the core structure, was required. An efficient synthesis of the carbon framework would then allow for versatile modification and preparation of natural product derivatives.

\section{Results and Discussion}

We focused our studies on the structural core motif (see figure 1a, marked in grey), which consists of a highly substituted central ring structure surrounded by additional rings including a cyclopentane and a $\gamma$-lactone. Specifically, our aim was to synthesize structural simplified analogs, investigate their neurite outgrowth activity and ultimately learn about the structure-activity relationship. Importantly, we have placed the emphasis on synthesizing simplified analogs, i.e. carbon framework derivatives of $( \pm)-\mathbf{1}$ and
( \pm -2. Two rationally simplified structural derivatives $\mathbf{4}$ and $\mathbf{5}$ (Figure 1b) were designed by altering the oxidation state and by targeted disconnection of hydroxo functionalities, e.g. oxetane and lactone moieties. The synthetic strategy towards these two derivatives is based on the rapid construction of the polycyclic structure from readily available enones. Retrosynthetically, structure 4 (Figure 1b) can be traced back to cyclopentenone 7 via ring disconnection to diketone $\mathbf{6}$. Analogously, lactone $\mathbf{5}$ is constructed from bicyclic ketone $\mathbf{9}$. Here, the $\gamma$-lactone ring is attached by employing the pre-functionalized enone 8

Synthesis of the core structure (depicted in Scheme 1) starts with commercially available dimethylcyclopentenone (10) which was subjected to an $\alpha$-methoxycarbonylation using Mander's reagent, ${ }^{[11]}$ followed by methylation of the 1,3-dicarbonyl with methyl iodide. A 1,4-addition of the in situ formed cuprate gave rise to 12 in excellent yield (96\%) and with a diastereoselectivity of 2.3:1. The two diastereomers could be separated via column chromatography. After Wacker-oxidation, the desired synconfiguration of diketone $\mathbf{1 3}$ was confirmed by X-ray crystal structure analysis. Attempts to close the cyclopentene ring failed under a variety of basic conditions. However, treatment with $p$ toluene sulfonic acid ${ }^{[12]}$ provided bicyclic ketone 14, along with conjugated iso-14 (r.r. = 2:1), which could be recycled via acidic equilibration. Finally, $\alpha$-alkylation with bromo alkyl acetate yielded structures 15 and $16(\mathrm{R}=\mathrm{Et}$ or $\mathrm{Me})$. The route described allows for the gram-scale access to diastereomerically pure carbon framework derivatives of the natural products.

Furthermore, we were interested in structure derivatives carrying the intact eastern lactone ring. Thus, alcohol $\mathbf{1 7}$ was found to be a suitable starting point. ${ }^{[13]}$ Functionalization with chloroformate and allylic oxidation with chromium(VI) oxide ${ }^{[14]}$ gave enone 18 in $48 \%$ yield over two steps. 1,4-Addition, Wacker-oxidation and acidic cyclization provided deconjugated 20 exclusively. Notably,
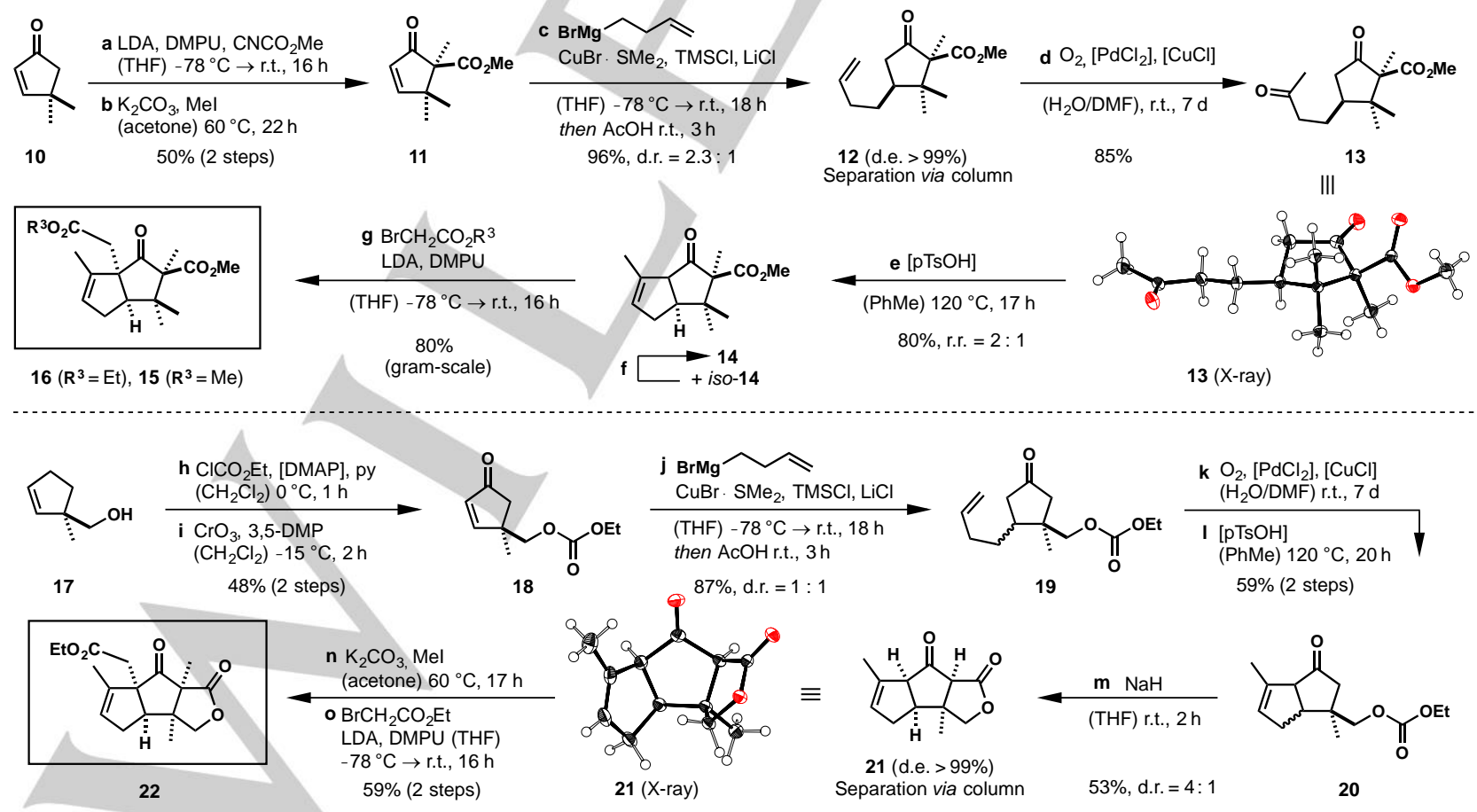

Scheme 1. Synthesis of the carbon frameworks 15,16 and $22 . \mathbf{f}[\mathrm{pTsOH}](\mathrm{PhMe}) 120^{\circ} \mathrm{C}, 17 \mathrm{~h}$, quant., r.r. $=2: 1$. LDA $=$ lithium diisopropylamide, DMPU $=4$-dimethylaminopyridine, $\mathrm{THF}=$ tetrahydrofuran, $\mathrm{TMSCl}=$ trimethylsilylchloride, $\mathrm{DMF}=\mathrm{N}, \mathrm{N}$-dimethylformamide, $\mathrm{DMP}=3,5$-dimethyl pyrazol. 
FULL PAPER

the carbonate group survived these transformations, serving not only as a protecting group for the primary alcohol, but also as building block and precursor for the lactone moiety, which was introduced by treatment with sodium hydride. At this point, the diastereomers could be separated via column chromatography and the desired syn-configuration of bowl-shaped 21 was confirmed by X-ray crystal structure analysis. The 1,3-dicarbonyl compound allowed for two selective $\alpha$-alkylations, first with potassium carbonate and methyl iodide and then using LDA and bromo ethyl acetate. The concise route provided access to the complete carbon framework structure $\mathbf{2 2}$, which resembles the $( \pm)$-Merrilactone A core structure including the eastern lactone ring.

With the carbon framework in hand, we turned our attention to further modifications of the core structure (see Scheme 2). Saponification of diester $\mathbf{1 6}$ under standard conditions $(\mathrm{KOH}$ in aqueous media) allowed for selective hydrolysis of the sterically more accessible ester to yield monoacid 23. However, the second ester group proved to be unreactive even under forcing conditions, presumably because of the steric bulk of the quaternary carbon center in $\alpha$-position. Furthermore, in case of successful formation of the $\beta$-keto acid, isolation of the product was thwarted by spontaneous decarboxylation. Ultimately, diacid $\mathbf{2 4}$ was accessible by employing potassium trimethylsilanolate and subsequent facile hydrolysis of the silyl ester.[15] For the investigation of the core structure at different oxidation levels, we turned our attention to reductive modifications of the framework. Unfortunately, a selective reduction of the carbonyl functionalities proved to be challenging. Only mixtures of different oxidation states or decomposed material were obtained with a variety of reagents (including borohydrides, aluminum hydrides or Bouveault-Blanc conditions $\left.{ }^{[16]}\right)$. However, triol 25 was accessible by employing lithium aluminum hydride reduction with careful choice of the work-up method. The configuration of the secondary alcohol, pointing outside of the bowl shape structure, was unambiguously confirmed by $\mathrm{X}$-ray crystal structure analysis.

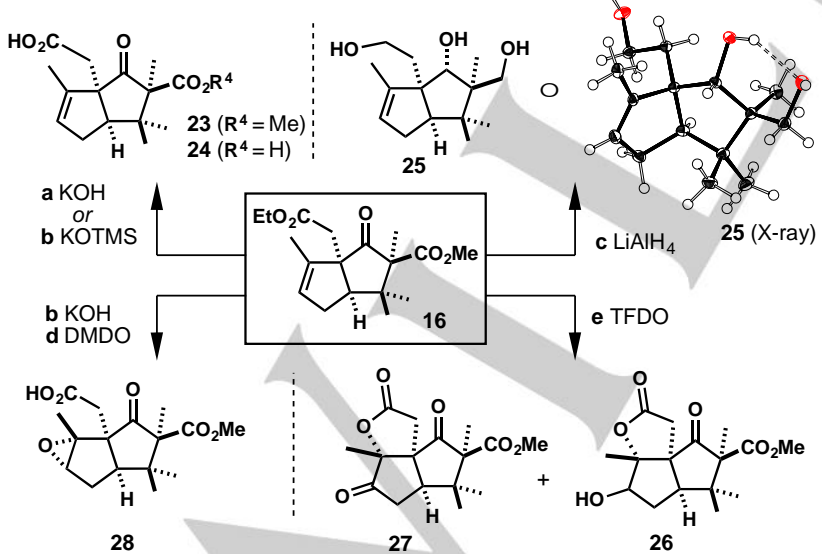

Scheme 2. Synthetic pathways starting from diester 16 to different derivatives of the natural product framework. a KOTMS (THF) r.t., 4-6 d, 85-95\%; b KOH, $\mathrm{H}_{2} \mathrm{O}(\mathrm{MeOH}) 70^{\circ} \mathrm{C}, 2 \mathrm{~h}, 90 \%$; c LAH, (THF) $-78 \mathrm{C} \rightarrow$ r.t., 18 h, 73\%; d DMDO, (ac) r.t., $2 \mathrm{~h}$, quant.; e TFDO (TFac) $-20^{\circ} \mathrm{C}, 17 \mathrm{~h}, 64 \%(1: 1)$. KOTMS = potassium trimethylsilanolate, $\mathrm{DMDO}=$ dimethyldioxirane, TFDO= trifluoromethyl(methyl)dioxirane; TFac = 1,1,1-trifluoroacetone.
Apparently, direction of the hydride by the primary $\beta$-alcohol group or preorganization by the hydrogen bonding (visible in the product's solid state structure) must have allowed for the hydride attack from the sterically more hindered concave side. In order to further vary the functionality of the olefin containing ring, we examined the epoxidation of ester 16 with TFDO. Surprisingly, the reaction yielded not the epoxide, but a mixture of lactones $\mathbf{2 6}$ and 27. These Anislactone-type cyclic structures were presumably formed by opening of the epoxide intermediate. The alcohol group formed was then further oxidized to the ketone under the reaction conditions. Notably, the use of milder DMDO yielded the desired epoxide 28, after saponification.

As depicted in Scheme 3, conversion of monoacid $\mathbf{2 3}$ with thionyl chloride, followed by reaction with aqueous ammonia and subsequent epoxidation with $m$-CPBA yielded amide 29 in $49 \%$ yield over two steps. X-ray structure analysis confirmed the structure and unequivocally proved the convex orientation of the epoxide. Carboxylic acid $\mathbf{2 3}$ was also exposed to oxidative conditions in order to increase the oxidation level of the carbon framework. Treatment with copper(II) acetate and hydrogen peroxide ${ }^{[17]}$ yielded allylic lactone 30 -an unprecedented product for these reaction conditions. Even more surprising was the formation of oxidation product $\mathbf{3 1}$ in the reaction of the epoxidized acid with ruthenium(II) chloride and potassium bromate. ${ }^{[18]}$ The product was apparently formed by carbon-carbon bond scission of the epoxide, subsequent oxidations and acetalization. Fortunately, a single crystal suitable for X-ray crystallography was obtained, which allowed structure elucidation of the unexpected product.

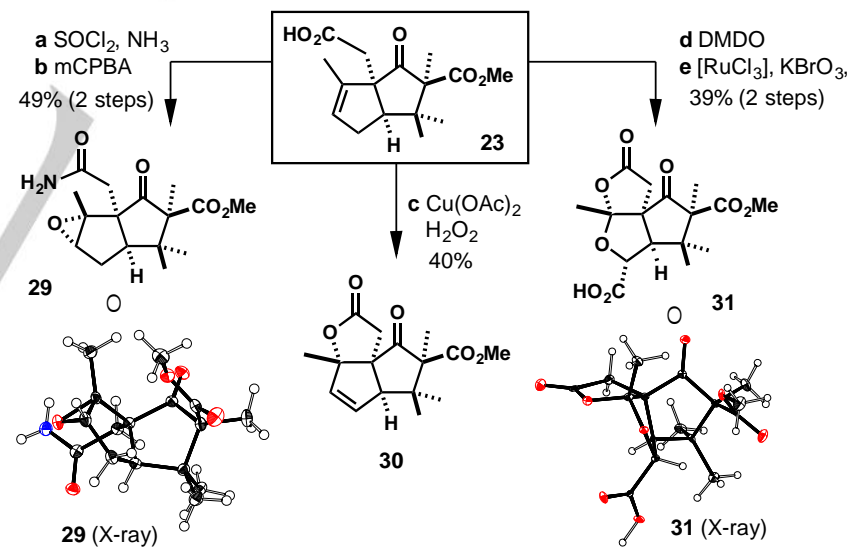

Scheme 3. Synthesis of epoxy amide 29, and unexpected oxidation products 30 and 31. a SOCl $2, \mathrm{NH}_{3}\left(\mathrm{CH}_{2} \mathrm{Cl}_{2}\right) 50{ }^{\circ} \mathrm{C}, 2 \mathrm{~h}, 74 \%$; b $m$ CPBA $\left(\mathrm{CH}_{2} \mathrm{Cl}_{2}\right)$ r.t., 18 h, $83 \%$; c $\mathrm{Cu}(\mathrm{OAc})_{2}, \mathrm{H}_{2} \mathrm{O}_{2}\left(\mathrm{MeCN} / \mathrm{H}_{2} \mathrm{O}\right)$ r.t. 1.4 h, $40 \%$; d DMDO (ac) r.t., 2 h, quant.; e [RuCl$l_{3}$, $\mathrm{KBrO}_{3}$, [py] ( $\left.\mathrm{MeCN} / \mathrm{H}_{2} \mathrm{O}\right) 60^{\circ} \mathrm{C}, 17 \mathrm{~h}, 39 \%$ (2 steps). mCPBA = meta-chloroperbenzoic acid, $\mathrm{DMDO}=$ dimethyldioxirane .

Additionally, amide derivatives of ester 15 were investigated (Scheme 4). After saponification, the amide group ${ }^{[19]}$ was introduced by coupling with EDC. Also in this case, the alkene could be epoxidized under mild conditions using DMDO. As it turned out, synthesis of diamide $\mathbf{3 2}$ was surprisingly challenging, presumably because of the steric hindrance of the $\alpha$-quaternary carbon center. All attempts to convert diacid $\mathbf{2 4}$ to the diamide failed to give difunctionalized product. However, after having tested a variety of conditions, it was found that direct treatment of 
the ester $\mathbf{1 5}$ with deprotonated amine allowed for conversion of both ester groups. By employing trifluoroacetic acid, diamide 32 could be further transformed to Anislactone-type structure 33, which was confirmed by $\mathrm{X}$-ray structure analysis.

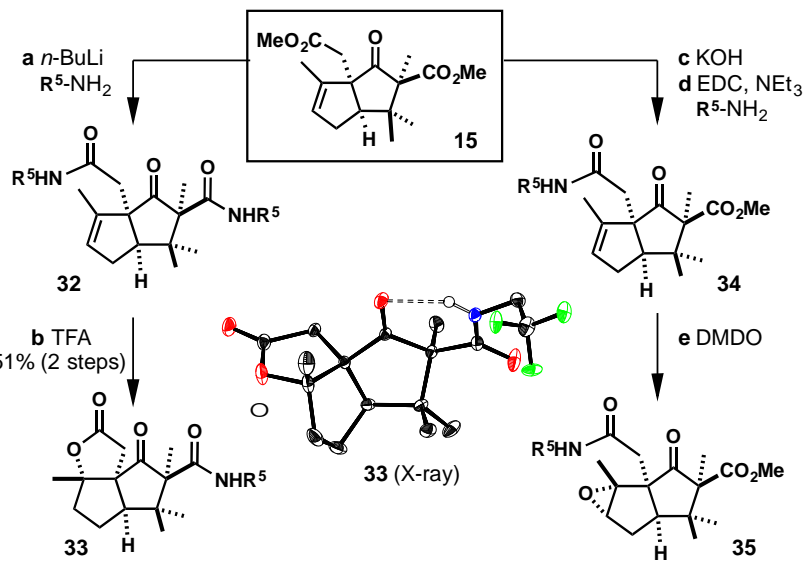

Scheme 4. Synthesis of amide derivatives. $\mathrm{R}^{5}=\mathrm{CH}_{2} \mathrm{CF}_{3}$. a $n$-BuLi, $\mathrm{R}^{5}-\mathrm{NH}_{2}$ (THF) $-78^{\circ} \mathrm{C}, 2$ h; b TFA $\left(\mathrm{CH}_{2} \mathrm{Cl}_{2}\right)$ r.t., 40 h, $51 \%$ (2 steps); c KOH, $\mathrm{H}_{2} \mathrm{O}(\mathrm{MeOH})$ $70^{\circ} \mathrm{C}, 2 \mathrm{~h}$; d EDC, R-NH $\mathrm{N}_{2}$, [DMAP], NEt $\left(\mathrm{CH}_{2} \mathrm{Cl}_{2}\right)$ r.t., 16 h, 29\% (2 steps); e DMDO, (ac) r.t., $14 \mathrm{~h}$, quant.. TFA = trifluoroacetic acid, EDC $=3$-(ethyliminomethyleneamino)- $N, N$-dimethylpropan-1-amine, $\mathrm{DMDO}=$ dimethyldioxirane.

In order to investigate the activity profile, we analyzed the effect of the prepared compounds on neuronal cells. Previous studies have shown that natural products of the illicium family and their structural analogs were capable of promoting NGF-mediated neurite outgrowth in neuronal cells (rat PC12 cells and primary cell cultures). $\left.{ }^{[6,}, 20\right]$ To explore the scope and speciesindependence of the neurotrophic activity of illicium sesquiterpenes, we here used mouse N2a cells-an established model for neurite outgrowth. ${ }^{[21 a-c]}$ We first validated the biological activity of a natural illicium sesquiterpene (synthetic Jiadifenolide $3^{[5 v][22]}$ in this model and found that it potently enhanced serum deprivation-induced neurite outgrowth $(140 \%$ compared to DMSO) with the strongest effect at a concentration of $1000 \mathrm{nM}$ (Figure 2 and data not shown). This magnitude of neurite outgrowth stimulations is comparable to the effect size of other neurotrophic agents in N2a cells ${ }^{[21 a-c]}$ as well as the effect of Jiadifenolide in other neurotrophic assays. ${ }^{[6 c, 20]}$ It is noteworthy that $\mathrm{N} 2 \mathrm{a}$ cells are not responsive to classical neurotrophins such as BDNF, suggesting that the neurotrophic effect of Jiadifenolide is independent of these mechanisms.

Since our synthetic approach has provided access to a variety of framework analogs, we next explored the biological profile of the synthetic derivatives. Figure 2 shows a graphical representation of the results of the biological activity study with the relative neurite outgrowth of the N2a cells in comparison to the control run (DMSO). An overview of the selected compounds and their activities is given in Table 1.

Figure 3 depicts representative images of selected N2a cells after neurite outgrowth in the presence of selected compounds. The prolonged dendrites are visible in case of neurotrophically active compounds (Figure $3 b, c, d$ ) relative to the control (Figure 3a, DMSO). Several compounds such as 15, 23 and 30 were identified that were almost as potent as (-)-Jiadifenolide, while being structurally highly simplified. A SAR analysis suggests that structural variations at the northern carboxy group are tolerated (methyl ester $\mathbf{1 5}$ or acid 23), while modifications of the western olefin reduce (compounds 27 and $\mathbf{3 1}$ ) or abolish activity (compounds $\mathbf{2 6}$ and 28). Likewise, derivatization of the eastern ester moiety abrogated activity (compare $23 \rightarrow \mathbf{2 4}$ and $\mathbf{1 6} \rightarrow \mathbf{2 2}$ ).

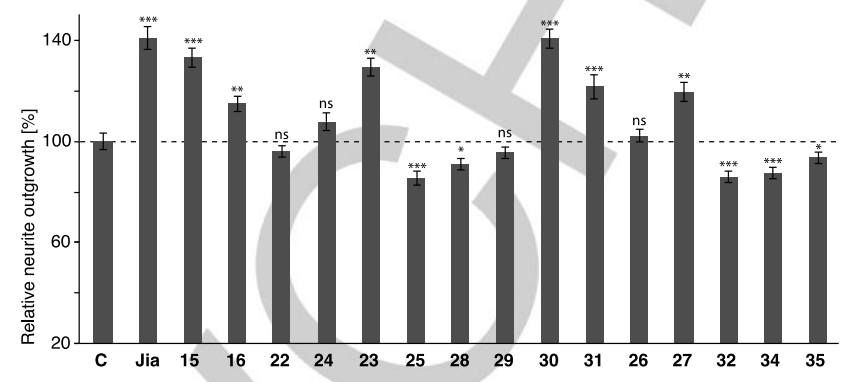

Figure 2. Relative neurite outgrowth of N2a-cells with selected compounds (at $1000 \mathrm{nM}$ concentration) in comparison to control (C, DMSO). Jia $=\mathbf{3}$, (-)-Jiadifenolide, concentration: $1000 \mathrm{nM}$. Data represent averages of three independent runs (analysis of $>25$ cells for each run). Error bars indicate \pm s.e.m. ${ }^{\star * *} \mathrm{P} \leq 0.001,{ }^{* *} \mathrm{P} \leq 0.01,{ }^{*} \mathrm{P}<0.05, \mathrm{~ns}=$ not significant.

The inactivity of $\mathbf{2 2}$ was surprising since this eastern lactone motif (ring $A$ in Figure 1) is present in Merrilactone $A$ and bioactive Jiadifenolide analogs. ${ }^{[6 c]}$ Furthermore, the distinct neurite outgrowth by lactone $\mathbf{3 0}(138 \%)$ is remarkable, considering that

Table 1. Overview of carbon framework derivatives and neurite outgrowth.

Compound (neurite outgrowth) ${ }^{a}$
C, DMSO (100\%) 3, (-)-Jiadifenolide (140\%)

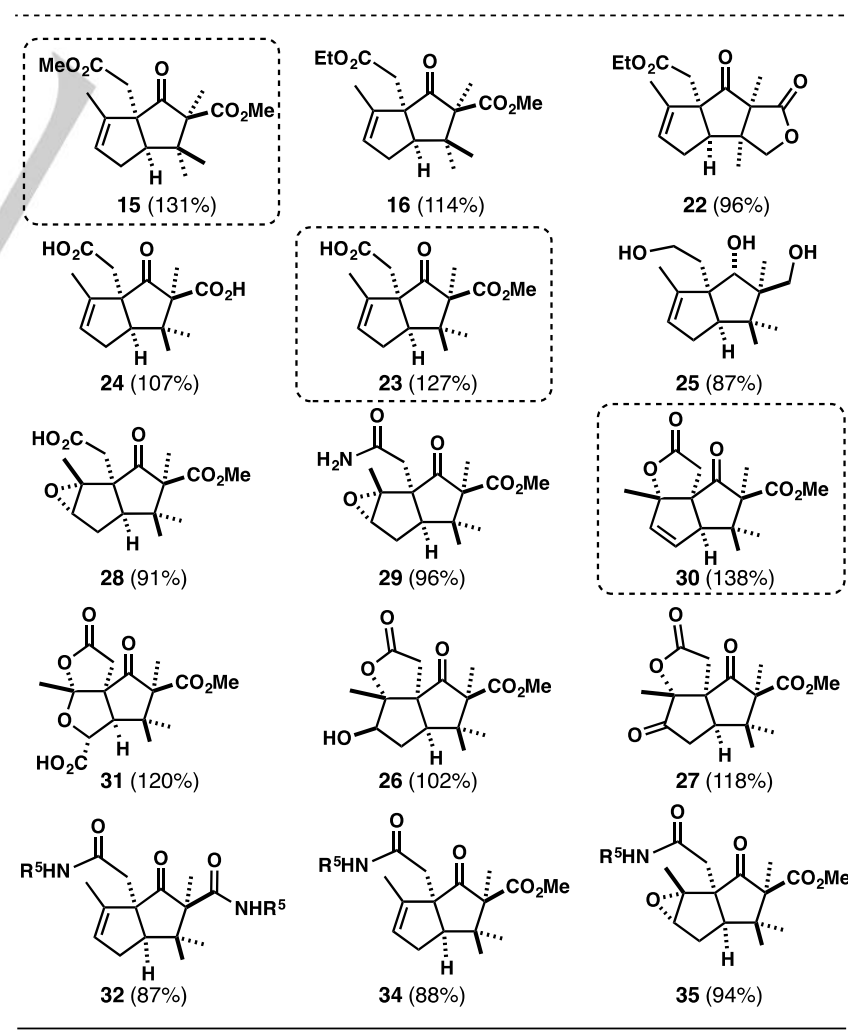

$\mathbf{R}^{5}=\mathrm{CH}_{2} \mathrm{CF}_{3}$, a) Relative neurite outgrowth [\%] in comparison to control (DMSO) 
WILEY-VCH

FULL PAPER

1

2

3

4

5

6

7

8

9

10

11

12

13

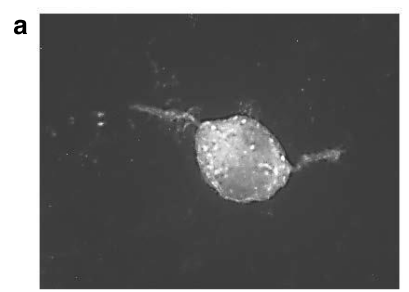

DMSO (control)

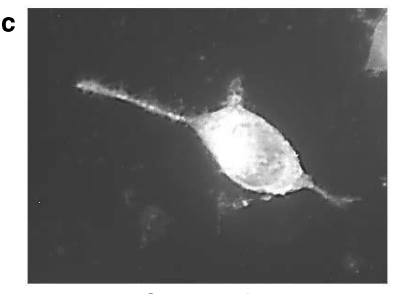

Compound 15

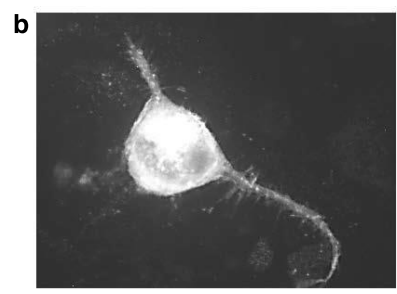

3, (-)-jiadifenolide

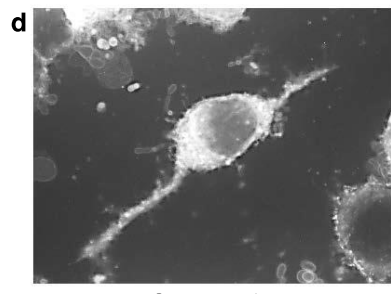

Compound $\mathbf{3 0}$
Figure 3. Representative images of N2a-cells after cell differentiation and neurite outgrowth. a DMSO (control); b (-)-Jiadifenolide (reference); c compound 15; d compound $\mathbf{3 0}$ (all at $1000 \mathrm{~nm}$ concentration).

the structure shows a high degree of similarity to the Anislactone structure, especially in light of the position of the $\gamma$-lactone moiety. Although it has been shown that Merrilactone A can be readily converted to the related natural product, ${ }^{[5 a]}$ to the best of our knowledge the biological activity of Anislactone A/B has not yet been validated. The results presented confirm that the natural product Merrilactone A can be structurally simplified while retaining biological activity. The most active derivatives $(\mathbf{1 5}, \mathbf{2 3}$, 30) are accessible in 6-8 synthetic steps with high yields from commercial starting materials. This compares favorably to the 1726 steps required for the total syntheses of $( \pm)$-Merrilactone $A$ reported.

\section{Conclusions}

In conclusion, a series of structural analogs derived from the neurotrophic illicium sesquiterpene natural products $( \pm)$-Merrilactone $A$ and $( \pm$ )-Anislactone $A / B$ has been synthesized. The concise synthetic route relies on the rapid construction of the carbon skeleton and enables the gram-scale preparation of the diastereomerically pure framework structure. Therefore, access is provided to further modified and functionalized analogs. In total, a library of 15 framework derivatives has been prepared, enabling the analysis of the structure-activity relationship. Our study identifies promising structural derivatives, i.e. simplified natural product analogs, which are accessible in only $6-8$ synthetic steps and still promote neurite outgrowth (138\% compared to control). These results will aid biochemical studies aimed towards elucidating the molecular mechanism and relevant targets underlying the neurotrophic activity of the illicium sesquiterpenes and analogs thereof. The simplified compounds could also facilitate the development of new pharmaceuticals for the treatment of neurodegenerative diseases.

\section{Acknowledgements}

This work was funded by the DFG - Deutsche Forschungsgemeinschaft (TI 831/2-1). Further support was provided by the TUM Junior Fellow Fund. We thank Prof. R. A. Shenvi for providing a sample of synthetic (-)-Jiadifenolide. Furthermore, the support of Prof. Thorsten Bach and his group (TUM) is acknowledged.

Keywords: Natural Products • Neurotrophic • Biological Activity - Synthesis $\cdot$ Sesquiterpenes

CCDC \#\#\#\#\# contains the supplementary crystallographic data for this paper (compounds 13, 21, 25, 29 and 33). These data can be obtained free of charge from The Cambridge Crystallographic Data Centre via www.ccdc.cam.ac.uk/structures.

\section{References}

[1] E. A. Crane and K. Gademann, Angew Chem Int Ed Eng/ 2016, 55, 3882 3902.

[2] J. Huang, R. Yokoyama, C. Yang and Y. Fukuyama, Tetrahedron Letters 2000, 41, 6111-6114.

[3] I. Kouno, K. Mori, N. Kawano and S. Sato, Tetrahedron Letters 1989, 30 7451-7452.

[4] M. Kubo, C. Okada, J. M. Huang, K. Harada, H. Hioki and Y. Fukuyama, Org Lett 2009, 11, 5190-5193.

[5] a) J.-M. Huang, C.-S. Yang, M. Tanaka and Y. Fukuyama, Tetrahedron 2001, 57, 4691-4698; b) V. B. Birman and S. J. Danishefsky, Journal of the American Chemical Society 2002, 124, 2080-2081; c) M. Inoue, T. Sato and M. Hirama, J Am Chem Soc 2003, 125, 10772-10773; d) K. Harada, H. Kato and Y. Fukuyama, Tetrahedron Letters 2005, 46, 7407-7410; e) J. Iriondo-

Alberdi, J. E. Perea-Buceta and M. F. Greaney, Org Lett 2005, 7, 3969-3971; f) G. Mehta and S. R. Singh, Tetrahedron Letters 2005, 46, 2079-2082; g) Z. Meng and S. J. Danishefsky, Angew Chem Int Ed Engl 2005, 44, 1511-1513; h) M. Inoue, T. Sato and M. Hirama, Angew Chem Int Ed Engl 2006, 45, 4843-4848; i) G. Mehta and S. R. Singh, Angew Chem Int Ed Eng/ 2006, 45, 953-955; j) K. Harada, H. Ito, H. Hioki and Y. Fukuyama, Tetrahedron Letters 2007, 48, 6105-6108; k) W. He, J. Huang, X. Sun and A. J. Frontier, J Am Chem Soc 2007, 129, 498-499; I) M. Inoue, N. Lee, S. Kasuya, T. Sato, M. Hirama, M. Moriyama and Y. Fukuyama, J. Org. Chem. 2007, 3065-3075; m) W. He, J. Huang, X. Sun and A. J. Frontier, J Am Chem Soc 2008, 130, 300308; n) L. Shi, K. Meyer and M. F. Greaney, Angew Chem Int Ed Engl 2010, 49, 9250-9253; o) J. Xu, L. Trzoss, W. K. Chang and E. A. Theodorakis, Angew Chem Int Ed Engl 2011, 50, 3672-3676; p) N. Nazef, R. D. Davies and M. F. Greaney, Org Lett 2012, 14, 3720-3723; q) J. Chen, P. Gao, F. Yu, Y. Yang, S. Zhu and H. Zhai, Angew Chem Int Ed Engl 2012, 51, 5897-5899; r) L. Trzoss, J. Xu, M. H. Lacoske and E. A. Theodorakis, Beilstein J Org Chem 2013, 9, 1135-1140; s) D. A. Siler, J. D. Mighion and E. J. Sorensen, Angew Chem Int Ed Engl 2014, 53, 5332-5335; t) I. Paterson, M. Xuan and S. M. Dalby, Angew Chem Int Ed Engl 2014, 53, 7286-7289; u) Y. Shen, L. Li, Z. Pan, Y. Wang, J. Li, K. Wang, X. Wang, Y. Zhang, T. Hu and Y. Zhang, Org Lett 2015, 17, 5480-5483; v) H. H. Lu, M. D. Martinez and R. A. Shenvi, Nat Chem 2015, 7, 604-607; w) J. Gomes, C. Daeppen, R. Liffert, J. Roesslein, E. Kaufmann, A. Heikinheimo, M. Neuburger and K. Gademann, J Org Chem 2016, 81, 11017-11034.

[6] a) D. A. Carcache, Y. S. Cho, Z. Hua, Y. Tian, Y. M. Li and S. J. Danishefsky, J Am Chem Soc 2006, 128, 1016-1022; b) D. Urabe and M. Inoue, Tetrahedron 2009, 65, 6271-6289; c) L. Trzoss, J. Xu, M. H. Lacoske, W. C. Mobley and E. A. Theodorakis, Chemistry 2013, 19, 6398-6408. [7] For recent reviews see: a) J. Xu, M. H. Lacoske and E. A. Theodorakis, Angew Chem Int Ed Engl 2014, 53, 956-987; b) R. A. Shenvi, Nat Prod Rep 2016, 33, 535-539.

[8] a) P. M. Joyner and R. H. Cichewicz, Nat Prod Rep 2011, 28, 26-47; b) P. Williams, A. Sorribas and M. J. Howes, Nat Prod Rep 2011, 28, 48-77.

[9] a) M. V. Sofroniew, C. L. Howe and W. C. Mobley, Annu Rev Neurosci 2001, 24, 1217-1281; b) E. J. Huang and L. F. Reichardt, Annu Rev Neurosci 2001, 24, 677-736; c) M. V. Chao, Nat Rev Neurosci 2003, 4, 299-309. 
[10] F. Longo, T. Yang, J. Knowles, Y. Xie, L. Moore and S. Massa, Current Alzheimer Research 2007, 4, 503-506.

[11] S. R. Crabtree, W. L. A. Chu and L. N. Mander, Synlett 1990, 1990, 169170

[12] G. Mehta and K. Pallavi, Tetrahedron Letters 2006, 47, 8355-8360.

[13] S. Pichlmair, M. de Lera Ruiz, K. Basu and L. A. Paquette, Tetrahedron 2006, 62, 5178-5194.

[14] W. G. Salmond, M. A. Barta and J. L. Havens, The Journal of Organic Chemistry 1978, 43, 2057-2059.

[15] E. D. Laganis and B. L. Chenard, Tetrahedron Letters 1984, 25, 58315834 .

[16] L. Bouveault and G. Blanc, Bull. Soc. Chim. France 1904, 31, 666-672.

[17] C. M. Rasik and M. K. Brown, Angew Chem Int Ed Eng/ 2014, 53, 1452214526.

[18] E. McNeill and J. Du Bois, J Am Chem Soc 2010, 132, 10202-10204.

[19] The trifluoroethyl amide group was employed as a directing group for $\mathrm{C}-\mathrm{H}$ functionalization J. Richers, M. Heilmann, M. Drees and K. Tiefenbacher, Org Lett 2016, 18, 6472-6475.

[20] M. Shoji, M. Nishioka, H. Minato, K. Harada, M. Kubo, Y. Fukuyama and

T. Kuzuhara, Biochem Biophys Res Commun 2016, 470, 798-803.

[21] a) S. Gaali, A. Kirschner, S. Cuboni, J. Hartmann, C. Kozany, G.

Balsevich, C. Namendorf, P. Fernandez-Vizarra, C. Sippel, A. S. Zannas, R.

Draenert, E. B. Binder, O. F. Almeida, G. Ruhter, M. Uhr, M. V. Schmidt, C.

Touma, A. Bracher and F. Hausch, Nat Chem Biol 2015, 11, 33-37; b) S.

Pomplun, Y. Wang, A. Kirschner, C. Kozany, A. Bracher and F. Hausch,

Angewandte Chemie International Edition 2015, 54, 345-348; c) S. Gaali, X.

Feng, A. Hähle, C. Sippel, A. Bracher and F. Hausch, Journal of Medicinal

Chemistry 2016, 59, 2410-2422.

[22] A sample of synthetic (-)-Jiadifenolide was kindly provided by Prof. Ryan Shenvi. 
1

2

3

4

5

6

7

8

9

10

\section{Entry for the Table of Contents}

\section{FULL PAPER}

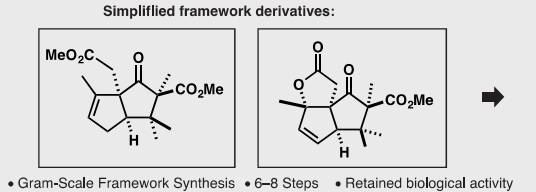

Gram-Scale Framework Synthesis 6 6-8 Steps - Retained biological activity

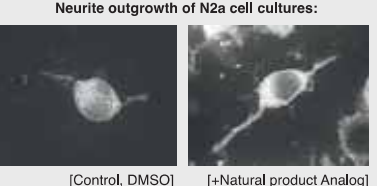

[Control, DMSO] It Natural product An:
Access to a series of structural analogs of the illicium sesquiterpene natural products $( \pm)$-Merrilactone $A$ and $( \pm)$-Anislactone $A / B$ is enabled by an efficient synthetic route. The derivatives can be prepared in 6-8 steps while retaining the biological activity as shown in neurite outgrowth studies.
Johannes Richers, Alexander Pöthig, Eberhardt Herdtweck, Claudia Sippel, Felix Hausch and Konrad Tiefenbacher* [Page No. - Page No.] Synthesis and Neurotrophic Activity Studies of Illicium Sesquiterpene Natural Product Analogs 